\title{
Asthma outbreaks: an opportunity for research?
}

Outbreaks of asthma typically consisting of up to hundreds of attendances at emergency departments by patients with unstable asthma, including one or two deaths within a few hours, in specific locations, have been repeatedly reported in the literature. Although respiratory physicians would readily accept the importance of studying both the occurrence and the aetiology of such outbreaks, other professionals and health authorities might not, especially in a context of limited resources.

Asthma is a common condition responsible for a high rate of morbidity and restricted activity. Its prevalence has increased in recent decades and both its causes and the reasons for its increasing prevalence are not well established. Outbreaks of asthma may provide an opportunity for identification of risk factors which are potentially preventable. From a public health point of view, outbreaks of asthma are seen within the context of avoidable morbidity and mortality. A particular outbreak of asthma, or even a series of outbreaks such as occurred in Barcelona with more than 1000 attendances at emergency departments and about 20 deaths, may appear unimpressive in comparison with the larger health burden imposed by many other diseases. In addition, the investigation of asthma outbreaks is difficult and usually requires a retrospective approach. Thus, it is relevant to question to what extent outbreaks of asthma should be investigated.

\section{Review of asthma outbreaks}

In 1928 Figley described a time and space cluster of asthma affecting about 200 patients living within a one mile radius of a castor bean mill in Toledo, Ohio, USA. The outbreak was caused by the inhalation of castor bean grinding dust and disappeared after the factory stopped processing castor beans. ${ }^{1}$ In the 1950 s outbreaks of asthma caused by exposure to castor beans were also reported in South Africa ${ }^{2}$ and Brazil. ${ }^{3}$

Series of asthma outbreaks have been reported both in New Orleans and in New York. ${ }^{45}$ The city of New Orleans experienced repeated outbreaks for nearly two decades, most of them involving about 100 attendances at the Charity Hospital emergency department. ${ }^{4}$ After considering several point source hypotheses it was postulated that the outbreaks resulted from sensitisation of the local atopic population to different seasonal aeroallergens. ${ }^{6} \mathrm{~A}$ subsequent decline in the occurrence of asthma epidemics was attributed both to better socioeconomic conditions and the availability of improved medical care for indigent patients. ${ }^{7}$ By contrast, outbreaks of asthma in New York were not spontaneously identified but were observed during an analysis to identify health indicators for air pollution studies. Although the epidemic days in New York fulfilled a well established statistical definition, they were smaller than the outbreaks mentioned above. No relation was found with air pollution and the days of high asthma incidence were more likely to coincide with Sundays, Mondays, Thanksgiving days, and the subsequent days, leading the authors to propose that exposure to indoor environmental agents might have been important. ${ }^{5}$ The days of high asthma incidence identified in New York probably reflect a tendency for time clustering of asthma, rather than individual clusters.

In 1985 Packe and Ayres reported an asthma outbreak involving about 80 patients treated in eight acute hospitals in Birmingham. ${ }^{8}$ This cluster coincided with a thunderstorm. The levels of chemical air pollution were low but there was a large increase in the levels of airborne fungal spores, especially Didymella exitialis and Sporobolomyces, around the time of the outbreak. ${ }^{9}$ A firm aetiological conclusion was not reached, and the association with the thunderstorm was not tested in a time series design. However, substantial information linking asthma with thunderstorms has been reported from Melbourne, Australia. Outbreaks of asthma coinciding with thunderstorms were first reported there by Morrison in $1960^{10}$ and by Egan in $1985 .^{11}$ More recently two further similar outbreaks were reported in Melbourne after thunderstorms, leading to the hypothesis that pollen grains are ruptured in rainwater by osmotic shock, each grain releasing about 700 starch granules small enough to enter the airways. ${ }^{12}$ The Melbourne researchers have shown that rye grass pollen grains contain a large quantity of starch granules which contain Lol $p$ IX and are coated with Lol p I. ${ }^{12}$ Twelve patients involved in the epidemic all had positive skin reactions to both standard rye grass pollen and isolated starch granules compared with seven of 13 outpatient controls with asthma $(p<0.05)$; four of the epidemic cases showed early bronchial constriction after voluntary re-exposure to starch granules. ${ }^{12}$ The levels of chemical air pollution on epidemic days were below or similar to the levels in a control period. ${ }^{12}$ While this is a relevant and well established hypothesis, sufficient aetiological evidence has not yet been provided. The association between thunderstorms and asthma in Melbourne, although likely to be real, is still anecdotal as it has also not been studied in a formal time series analysis with complete ascertainment of epidemic days. The case control study included only 12 of the epidemic asthma cases from one single hospital out of the 300 or so patients affected, and the controls were selected from an outpatient department where the association between epidemic asthma and starch allergy was not adjusted for potential confounders such as atopy.

Another outbreak of asthma following a thunderstorm has recently been reported in London. ${ }^{13}$ The preliminary data suggest that the increased admissions to emergency departments for asthma totalled about 1000 in a period of two days, possibly the largest outbreak ever recorded ${ }^{13}$ (K Venables, personal communication). The coincidence of outbreaks of asthma with thunderstorms allows the Birmingham and Melbourne hypotheses to be examined again, and a large multidisciplinary study group has been established.

In Barcelona five outbreaks of asthma were reported from a single hospital (Hospital Clinic) between 1981 and 1983. The potential link with air pollution and local public health interest allowed a collaborative group to be established with the aims of long term research and follow up. ${ }^{14}$ The asthma outbreaks typically showed a point source distribution and, after rejecting several hypotheses, a possible link with unloading of soybean at the city harbour was established. A causal relation between the unloading of soybean and asthma outbreaks was subsequently established through a time series study of harbour activities, a serum case control study, and an intervention study. ${ }^{15-17}$ The results of these studies led to the identification of similar outbreaks caused by unloading of soybean in Car- 
tagena, Spain. ${ }^{18}$ More recently Italian researchers have investigated an outbreak of asthma in Naples which affected more than 100 patients in a single day. The outbreak coincided with the presence of a large ship unloading soybean in Naples harbour and about $50 \%$ of those affected had IgE antibodies to soybean. ${ }^{19}$ Recently, White et al have shown that outbreaks of asthma in New Orleans were more likely to occur after a ship carrying soybean arrived in the harbour and, in particular, on the following day, ${ }^{20}$ thus supporting the suggested association between asthma and soybean in Barcelona.

Several conclusions may be derived from this brief review. Firstly, asthma can occur in epidemic form usually as short time and space clusters. Secondly, with the exception of the days of high incidence of asthma in New York, the large number of cases involved in the outbreaks and their severity led to spontaneous identification of the outbreaks by physicians. Thirdly, although soybean and castor bean are well established causes of outbreaks of asthma, for most outbreaks sufficient aetiological evidence has not yet been provided.

\section{An opportunity for research}

The most important reason for investigating outbreaks of asthma is the substantial public health burden which they can impose on a community. The aim of research is the identification of a cause and its potential prevention. Repeated outbreaks are more likely to promote a local research effort which may involve evaluation by the local health authorities and an appreciation of the potential cost may be advisable. In the case of the research into the asthma outbreaks in Barcelona an economical study showed that the benefits of the investigation exceeded their cost (Murillo $\mathrm{C}$, personal communication).

It may happen that local outbreaks are an index of other previously unrecognised outbreaks. In occupational asthma the identification of outbreaks has frequently been followed by reports of similar previously unidentified cases. This has also been the case in Barcelona where the soybean hypothesis was further corroborated in Cartagena, Spain and probably in New Orleans and Naples. The same could occur if the Melbourne hypothesis is corroborated in London. In such cases the benefits of investigating local outbreaks are likely to go beyond just the local community.

There are other valid reasons for investigating asthma outbreaks, since they are natural experiments in which the epidemiological approach can establish a strong time order sequence between an exposure and its outcome. Time order is a relevant criterion of causality. Most diseases result from constellations of component causes, each one in itself being insufficient to produce the disease. However, as Knox has pointed out, when an outbreak occurs the cases are related to each other through some social or biological mechanism or have a common relation to some other event or circumstance. ${ }^{21}$ In the Barcelona outbreaks this common event was the inhalation of soybean dust which constitutes a necessary, although insufficient, cause. The assessment of a common factor used to show a strong association with an epidemic which is stronger as the prevalence of an individual factor is lower in the reference group. The strength of an association is considered another important criterion of causality. In addition, if an aetiological cause is found then other relevant risk factors may be properly assessed. With this approach we have reported an interaction between atopy and smoking in soybean epidemic asthma, ${ }^{22}$ reinforcing the evidence of a relation between smoking, occupation, and allergic asthma from occupational studies. ${ }^{2324}$ Another example of a risk factor that could be assessed in a natural model of epidemic asthma is air pollution. An experimental study suggesting that ozone may potentiate allergic bronchial responsiveness $^{25}$ led us to test whether this was the case in the Barcelona outbreaks. Although preliminary analysis suggested that the probability of an asthma outbreak was higher on days with higher levels of air pollution, ${ }^{26}$ further analysis has been inconclusive (unpublished data) and the hypothesis is still being tested. Other asthma outbreaks may provide a good opportunity to assess possible synergism between air pollution and allergens within the general level in a population.

Some negative considerations should also be stated. The investigation of clusters of cancer has shown that it may be very difficult to define statistically a cluster and that rare diseases tend to cluster. Some authors have stressed the risk of spending resources on investigating apparent clusters that ultimately are due to chance or to the uneven distribution of risk factors both in time and space (responsible for clustering). Rothman has argued that the study of individual clusters of disease do not offer hopeful prospects for scientific advance. ${ }^{27}$

\section{How to approach the study of asthma outbreaks}

The problems surrounding the investigation of clusters led the Centers for Disease Control to elaborate a set of guidelines that may also be taken as a reference. ${ }^{28}$ These guidelines have proved useful in dealing with clusters of several diseases including cancer and congenital malformations. The protocol includes the following steps: first contact, preliminary evaluation, major feasibility study, major aetiological studies, intervention. An important step in this protocol is the transition from the preliminary evidence to major aetiological studies. The authors suggest considering the following three criteria - high incidence rate, high exposure documented, and biological plausibility - and taking the presence of two of them as an indication of the need for complete aetiological investigation. Most of the outbreaks of asthma reviewed above included evidence of a high incidence rate (usually tenfold increase) and biological plausibility of particular aeroallergens being involved and are, in consequence, suitable for further investigation.

The investigation of the soybean asthma outbreaks in Barcelona suggests that the following steps, if applied flexibly, may be of help: (1) descriptive studies within well defined time/space coordinates to estimate the magnitude of the outbreak and to search for a potential time/space pattern as described by $\mathrm{Knox}^{29}$; (2) a process of hypothesis generation; (3) complete aetiological studies when a firm hypothesis is available; (4) prevention or prediction if a suitable factor has been implicated followed by intervention studies if an intervention has been implemented; and (5) clinical epidemiological and mechanistic studies on epidemic asthma for specific issues of scientific relevance.

After decades of lethargy the interest in investigating outbreaks of asthma has steadily increased. It is unclear whether this results merely from the pendular swing of our scientific interests or whether it is because of an increasing frequency of asthma outbreaks in parallel with an increasing prevalence of asthma. Whatever the case, when an outbreak of asthma of small or moderate intensity is suspected or has occurred, its investigation should not be simplistically overstated or dismissed. Indeed, the need for further research should be systematically evaluated, weighing its potential public health and scientific interest against the methodological difficulties and cost. When a large outbreak of asthma occurs, and especially when apparently similar asthma outbreaks occur repeatedly within the same population, the available evidence suggests that they deserve 
further investigation until firm aetiological evidence is established.

The author wishes to thank Dr Kate Venables for her valuable comments. The author was Visiting Principal Fellow at the National Heart and Lung Institute Royal Brompton Hospital, London, UK, in receipt of a grant from Fondo de Investigacion Sanitaria (94/5838), Spain.

\section{Department of Epidemiology}

and Public Health,

JOSEP M ANTO

Institut Municipal d'Investigacio Medica,

Universitat Autonoma de Barcelona,

Dr Aiguader 80,

08003 Barcelona

Spain

1 Figley $\mathrm{KD}$, Elrod RH. Endemic asthma due to castor bean dust. $\mathcal{F} A M A$ 1928;90:79-82.

2 Mendes E, Cintra U. Collective asthma, simulating an epidemic, provoked by castor-bean dust. F Allergy 1954;25:253-9.

3 Ordman D. An outbreak of bronchial asthma in South Africa, affecting more than 200 persons, caused by castor bean dust from an oil-processing more than 200 persons, caused by casto

4 Salvaggio JE, Klein RC. New Orleans asthma I. Characterization of individuals involved in epidemics. $\mathcal{f}$ Allergy 1967;39:227-34.

5 Goldstein IF, Cuzick J. Daily patterns of asthma in New York City and in New Orleans: an epidemiological investigation. Environ Res 1983;30: 211-23.

6 Salvaggio J, Seabury J, Schoenhardt A. New Orleans asthma V. Relationship between Charity Hospital admission rates, semiquantitative pollen and fungal spore counts, and total particulate aerometric sampling data. $\mathcal{F}$ Allergy Clin Immunol 1971;48:96-114.

7 Goldstein IF, Salvaggio J. The decline of New Orleans asthma epidemics. Rev Environ Health 1984;4:133-46.

8 Packe GE, Ayres JG. Asthma outbreak during a thunderstorm. Lancet 1985; ii: $199-204$.

9 Packe GE, Ayres JG. Aeroallergen skin sensitivity in patients with severe asthma during a thunderstorm. Lancet 1986;: $850-1$

10 Morrison I. It happened one night. Med F Aust 1960;1:850-1.

11 Egan P. Weather or not. Med f Aust 1985;142:330.
12 Suphioglu C, Singh MB, Taylor P, Bellomo R, Holmes P, Puy R, et al. Mechanism of grass-pollen-induced asthma. Lancet 1992;339:569-72. Murray V, Venables K, Laing-Morton T, Partridge $M$, Williams D. Epidemic of asthma possibly related to thunderstorms. BMf 1994;309:131-2.

14 Antó JM, Sunyer J and Asthma Collaborative Group of Barcelona. A pointsource asthma outbreak. Lancet 1986;i:900-3.

15 Antó JM, Sunyer J, Rodriguez-Roisin R, Suarez-Cervera M, Vazquez L and the Toxicoepidemiological Committee. Community outbreaks of asthma associated with inhalation of soybean dust. $N$ Engl f Med 1989;320 1097-102.

16 Sunyer J, Antó JM, Rodrigo MJ, Morell F. Case-control study of serum immunoglobulin-E antibodies reactive with soybean in epidemic asthma. Lancet 1989;i:179-82.

17 Antó JM, Sunyer J, Reed ChE, Sabrià J, Martinez F, Morell F, et al. Preventing asthma epidemics due to soybeans by dust-control measures. N Engl ₹ Med 1993;329:1760-3.

18 Navarro C, Márquez M, Hernando L, Galvañ F, Zapatero L, Caravaca F. Epidemic asthma in Cartagena, Spain, and its association with soybean sensitivity. Epidemiology 1993;4:76-9.

19 D'Amato G, Liccardi G, Cazzola M. Environment and development of respiratory allergy. I. Outdoors. Monaldi Arch Chest Dis 1994;5:406-11.

20 White MC, Etzel RA, Olson DR. Reexamination of epidemic asthma in New Orleans in relation to the presence of soy-carrying ships in the harbor. Am 7 Epidemiol 1993;138:607.

21 Knox EG. Detection of clusters. In: P Elliott, ed. Methodology of enquiries into disease clustering. London: Small Area Health Statistics Unit, London School of Hygiene and Tropical Medicine, 1989.

22 Sunyer J, Anto JM, Sabria J, Rodrigo MJ, Roca J, Morell F, et al. Risk factors of soybean epidemic asthma: the role of smoking and atopy. $\mathrm{Am}$ Rev Respir Dis 1992;145:1098-102.

23 Venables KM, Dally MB, Nunn AJ, Stevens JF, Stephens R, Farrer N, et al. Smoking and occupational allergy in workers in a platinum refinery. BMF 1989;299:939-42.

24 Smoking, occupation and allergic lung disease (editorial). Lancet 1985;i: 965.

25 Molfino NA, Wright SC, Katz I, Tarlo S, Silverman F, McClean PA, et al. Effect of low concentrations of ozone on inhaled allergen responses in asthmatic subjects Lancet 1991;338:199-203.

26 Castellsague J, Sunyer J, Saez M, Murillo C, Anto JM. Effect of air pollution in asthma epidemics caused by soybean dust [abstract]. Eur Respir F 1992; 5(Suppl 15): 413S

27 Rothman KJ. A sobering start for the cluster busters' conference. $A m \mathcal{F}$ Epidemiol 1990;132:S6-13.

28 Knox EG. Epidemics of rare diseases. Br Med Bull 1971;27:43-7.

29 CDC. Guidelines for investigating clusters of health events. Morbidity and Mortality Weekly Report 1990;39:1-23. 